\title{
La qualité au Parlement
}

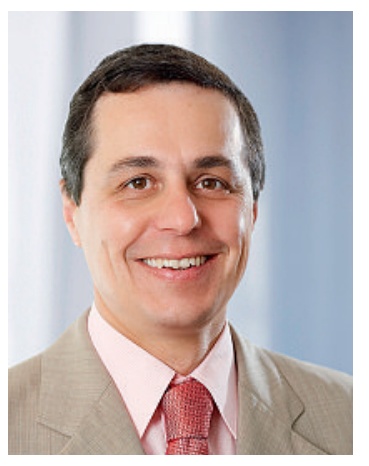

La réforme de la LAMal 09.053, appelée «Mesures urgentes pour endiguer les coûts de la santé» et lancée par le Conseil fédéral (CF) en mai 2009 pour répondre à l'augmentation des primes, est tombée à l'eau. Par 97 voix contraires et 76 favorables, l'alliance PS et UDC - avec l'abstention des Verts - a écrasé au Conseil national $(\mathrm{CN})$ cette mini-réforme. La logique de ce virage n'a que peu à voir avec le contenu de la réforme: le socialiste valaisan Stéphane Rossini a annoncé à la surprise générale que son groupe n'allait pas soutenir cette réforme. L'UDC a interprété ce virage comme une tactique pour maintenir la pression sur le système de santé (en vu du lancement de l'initiative populaire pour une caisse unique fédérale et publique) et pour se profiler comme les seuls avocats des patients. Le groupe UDC a alors immédiatement appuyé le bouton rouge et balayé ainsi - en 20 secondes - le travail d'une année et demie. Vive la Suisse!

Et pourtant la modification avait des propositions intéressantes, comme le nouvel article sur la qualité [1]. La qualité demeure en effet un thème important, tant au niveau professionnel que politique. Elle est un peu comme la Bible: tout le monde la cite, mais très peu l'ont lue. Sa définition reste floue et elle se laisse facilement instrumentaliser pour d'autres objectifs.

\section{Plusieurs actes parlementaires deman- dent un institut national de la qualité}

Indépendamment de ce paquet de mesures urgentes... et rejetées, qu'en est-il d'ailleurs de la qualité dans le système de santé? Elle a été explicitée dans la loi pour la première fois en 1996 (art. 58), avec l'entrée en vigueur de la LAMal. Dans un esprit libéral, le législateur avait alors délégué aux partenaires tarifaires (médecins et assureurs) le soin de définir bilatéralement les procédures de qualité: il voulait leur laisser pleine liberté de manœuvre. Dix ans plus tard, le Parlement s'est interrogé sur l'état d'avancement des travaux et a mandaté la Commission de la gestion pour évaluer la situation. Le 13 novembre 2007, cette dernière a publié le rapport «Evaluation du rôle de la Confédération dans la garantie de la qualité selon la LAMal» [2] se basant sur l'expertise effectuée par le Contrôle parlementaire de l'administration «Rôle de la Confédération dans la garantie de la qualité selon la LAMal» [3] du 5 septembre 2007. Conclusion: la situation est insatisfaisante! La qualité est gérée de manière fragmentée, discontinue, et sans vision d'ensemble. Le Parlement a alors décidé d'activer le rôle subsidiaire de l'Etat et de confier au CF le leadership en matière de qualité. Dans son avis du 18 juin 2008, le CF s'est déclaré prêt à l'assumer [4]. Pour ce faire, il a présenté le 9 octobre 2009 sa stratégie globale en matière de qualité [5]. Depuis lors - par le biais de l'OFSP -, il travaille à sa réalisation avec plusieurs acteurs, notamment la FMH. En même temps, différents actes parlementaires ont été déposés: l'initiative parlementaire Heim (07.486), transformée par la Commission de la santé du CN (CSSS-N) en motion (10.3015); la motion CSSS-E (Sommaruga, 10.3353) et la motion du groupe libéral-radical (Cassis, 10.3450). Le 28 septembre 2010, le CN a largement accepté la motion 10.3015 , soutenue entretemps même par le CF. Tous ces actes parlementaires demandent la création d'un institut national de la qualité qui puisse piloter, coordonner et unifier l'ensemble des efforts en cours. Cet institut est donc en préparation.

\section{L'acronyme HTA entre peu à peu dans le vocabulaire politique}

A côte de la qualité, l'économicité des prestations médicales (actes, médicaments, appareillage, etc.) est de plus en plus débattue. L'acronyme HTA (Healh Technology Assessment ou Medical board) entre peu à peu dans le vocabulaire politique. La motion du groupe libéral-radical (Cassis, 10.3451) demande l'institution d'une agence nationale dédiée au HTA. Sur cette motion, le Parlement n'a pas encore tranché, alors que le CF s'y est déclaré favorable. Il est fort à parier que le HTA sera prochainement un acronyme «à la mode», à côté de la qualité. Reste à faire le lien entre monde politique et monde professionnel, pour éviter que ces thèmes se développent sur deux voies parallèles qui s'ignorent ou, pire, se combattent! Le département DDQ de la FMH travaille avec beaucoup de passion et d'énergie pour assurer ce lien.

\section{Dr Ignazio Cassis Vice-président de la FMH et Conseiller national}

P.S. Pour approfondir: copiez les codes cités dans le champ «Recherche» du site www.parlement.ch!

1 Art. $436^{\text {bis }}$ (nouveau)

Les assureurs peuvent prévoir une valeur du point tarifaire plus élevée ou une prime de qualité dans les conventions conclues avec des fournisseurs de prestations dont la qualité du travail est régulièrement contrôlée par un organisme de certification accrédité.

2 FF no 38, pages 7089-7092 (www.admin.ch/ch/f/ff/2008/).

3 FF n ${ }^{\circ} 38$, pages 7093-7182 (www.admin.ch/ch/f/ff/2008/).

4 FF n ${ }^{\circ} 39$, pages 7183-7190 (www.admin.ch/ch/f/ff/2008/).

5 http://bit.ly/cTXIzX 